\title{
DETERMINANTES DO INVESTIMENTO DIRETO JAPONÊS NO BRASIL NA DÉCADA DE 80: UMA ANÁLISE EMPÍRICA ${ }^{1}$
}

Eduardo Kiyoshi Tonooka ${ }^{2}$

RESUMO: A visão dominante sobre o investimento direto estrangeiro (IDE) japonês no setor manufatureiro, baseada no argumento da relocalização de indústrias em declínio no Japão para países com menor nível de desenvolvimento industrial, foi adequada para explicar a maior parte do investimento japonês ao exterior, inclusive ao Brasil, até o final dos anos 70. As novas situações vivenciadas pelo Japão na década de 80 , no entanto, passaram a exigir novas explicações para a dinâmica do IDE japonês, particularmente no caso brasileiro.

A partir da avaliação das contribuições teóricas e das pesquisas empíricas existentes para a compreensão do IDE japonês, desenvolveu-se um modelo econométrico com o objetivo de identificar os principais determinantes do investimento direto japonês no Brasil na década de 80. Este modelo apresenta a particularidade de incorporar dados sobre as firmas que investem no país, procedimento pouco usual nos trabalhos empíricos sobre o tema que, na sua grande maioria, restringem-se aos dados setoriais.

ABSTRACT: The prevailing view about Japanese foreign direct investment (FDI) in the manufacturing sector based on the argument that it has suited to the reallocation of sunset industries in Japan to countries with lower levels of industrial development was adequate for explaining the majority of Japanese investment overseas up to late 70 s.

1. Este trabalho é baseado no capítulo 5 da tese de doutorado do autor intitulada "Investimento Direto Japonês na Década de 80: Uma Análise dos seus Determinantes no Brasil e no Mundo" O autor agradece à Fundação Japão e à Fapesp pelo apoio financeiro à realização deste trabalho.

2. Pesquisador-Visitante do Instituto de Pesquisas em Economia e Administração da Universidade de Kobe. 
Nevertheless, the new situations that faced Japan in the 80s demanded new interpretations for the dynamics of Japanese FDI, specially for the Brazilian case.

After evaluating the available theoretical contributions and empirical research for understanding Japanese FDI, an econometric model was developed in order to identify the main determinants of Japanese direct investment in Brasil in the 80s. This model embodies firm level data, as opposed to most empirical work on FDI motives which deals with industry level data.

PALAVRAS-CHAVE: Economia, investimento direto, investimento japonês, modelo econométrico, ativos específicos.

KEYWORDS: Economics, direct investment, Japanese investment, econometric model, specific assets.

\section{Introdução}

Até a década de 70, os momentos de maiores influxos de investimentos diretos japoneses ao Brasil estiveram diretamente associados à condução das políticas de desenvolvimento industrial por parte do governo brasileiro. Durante a década de 50, investimentos japoneses de grande porte foram realizados especialmente na vigência do Plano de Metas, quando instalaram-se no país empresas japonesas dos setores têxtil, siderúrgico, de construção naval e de máquinas leves. A partir da segunda metade dos anos 60 em diante, muitas pequenas e médias empresas japonesas dirigiram-se ao Brasil para fugir às restrições na oferta de mão-de-obra e de recursos naturais em curso no Japão. Na década seguinte, as relações com o governo brasileiro se estreitaram com a realização em conjunto de uma série de projetos nas áreas extrativa-mineral, petroquímica, siderúrgica, metalúrgica e de papel e celulose. A esses anos de auge nas relações entre os dois países, seguiu-se um longo período de esfriamento, iniciado com a crise da dívida externa, em 1982. Mais de dez anos depois, um novo clima de otimismo nas relações econômicas bilaterais pareceu ressurgir, na medida em que se consolidava a estabilidade monetária e ampliavam-se as oportunidades de investimento no Brasil após o Plano Real. Entretanto, contrariamente ao que fizeram Estados Unidos e alguns países da União Européia, o Japão investiu relativamente pouco na segunda metade da década de 90 , fato que acabou não configurando um novo ciclo de investimentos japoneses no país.

\section{Determinantes do Investimento Direto Japonês no Brasil na Década de 80: Uma Análise Empírica}

O estudo econométrico aqui desenvolvido reconhece que a identificação dos determinantes do investimento direto japonês no Brasil abrange três diferentes níveis de análise: 
a. nivel "macro": avaliar a importância de variáveis macroeconômicas sobre o investimento direto japonês no Brasil, fundamentalmente, relacionadas ao tamanho e potencial de crescimento do mercado interno e à política econômica oficial;

b. nível "meso" ou setorial: avaliar a importância das características de cada indústria sobre o investimento direto japonês no Brasil, incluindo estrutura de oferta (concentração industrial) e de produção (utilização intensiva de mão-deobra e/ou de recursos naturais), custos relativos de produção (especialmente, os associados à contratação de mão-de-obra), tamanho e potencial de crescimento do mercado;

c. nível "micro" ou da firma: avaliar a importância das características internas à firma sobre o investimento direto de cada firma no Brasil, como os gastos com pesquisa e desenvolvimento (P\&D), que representam a capacidade da firma em desenvolver bens intermediários, tais como tecnologia, reputação e organização gerencial, e os gastos com propaganda, que refletem a capacidade da firma em diferenciar seus produtos. Mais do que gastos, esses dispêndios configuram investimentos para a criação dos ativos específicos da firma.

\subsection{Definição da variável dependente e do método de estimação}

A complexidade e a variedade presentes na análise do investimento direto estrangeiro permitem abordá-lo segundo diferentes metodologias, sob o ponto de vista empírico, que dependem do foco dado ao fenômeno. Num primeiro momento, poderse-ía afirmar que os trabalhos quantitativos sobre investimento direto de um país $\mathrm{A}$ para um país B deveriam partir de sua definição para a construção da variável dependente, assumindo-a como sendo a soma dos valores monetários remetidos pelas firmas com sede no país A para suas afiliadas no país B (participação no capital social das afiliadas e empréstimos intercompanhias) e do montante de lucros reinvestidos nessas afiliadas.

Desconsiderando-se a disponibilidade dos dados acima ou do papel a ser desempenhado pelo reinvestimento (e também pelos recursos para investimento captados no mercado financeiro dos próprios países em que estão instaladas as afiliadas ou em terceiros mercados), dependendo do tipo de investigação, pode ser necessário partir-se de um ponto de partida diferente. Se, por exemplo, interessa avaliar o impacto das empresas estrangeiras de uma determinada nacionalidade sobre a economia de um país hospedeiro em termos de valor adicionado à produção, empregos gerados, impostos recolhidos, ou o que seja, a medida da participação dessas empresas pode ser conseguida através de outras definições.

Neste trabalho, pretende-se identificar os determinantes da presença das firmas japonesas no Brasil e a importância relativa das suas atividades no país. Dado que o período de análise corresponde à década de 80 , não apenas as empresas que iniciaram suas atividades no país nessa época, mas também as que se estabeleceram antes, são consideradas.

A importância relativa das atividades no Brasil para uma firma japonesa seria quantificada, idealmente, através de uma medida das expectativas da firma quanto ao 
retorno sobre o investimento realizado ou a realizar, estando, portanto, refletidas nas intenções de investimento ou de desinvestimento no país em relação às intenções de investimento global. Eventualmente, além do investimento direto, deveriam ser levados em consideração os recursos captados no mercado financeiro brasileiro ou em terceiros mercados, bem como os fluxos de serviços entre matriz e afiliadas (direitos de uso sobre tecnologia, capacidade de marketing, envio de executivos etc.), pois também caracterizam o relacionamento entre essas empresas.

Entretanto, as expectativas de retorno não são observáveis, ou mensuráveis, diretamente, enquanto que as intenções de investimento relativas são de difícil levantamento. Assim, tendo em vista a dificuldade para se obter determinadas informações e a disponibilidade dos dados, estabeleceu-se como variável dependente, para mensurar a importância relativa das atividades no Brasil para uma firma japonesa, a razão entre o número de empregados das afiliadas de uma determinada firma japonesa no Brasil e o número de empregados de sua matriz no Japão. Neste caso, assume-se que exista uma associação positiva entre a importância relativa das atividades em um certo país e o número relativo de empregados mantidos nesse mesmo país para uma firma qualquer. Dentre os fatores que podem comprometer essa associação estão a utilização de tecnologias de produto e de processo muito diferentes entre matriz e afiliadas e divergências quanto às atividades desempenhadas na matriz e nas afiliadas.

Evidentemente, da forma como foi definida, a variável dependente não pode assumir valores negativos, muito embora as expectativas de retorno ou as intenções de investimento possam. Desta maneira, a partir dessa variável não seria possível diferenciar uma firma que não investe no Brasil (intenção de investimento igual a zero) de outra que realiza um desinvestimento total (intenção de investimento menor que zero), pois ambas seriam caracterizadas pelo valor nulo para a variável dependente. A censura sobre a variável dependente, que em princípio também pode apresentar um número substancial de valores iguais a zero, requer um método de estimação mais sofisticado que o de mínimos quadrados. Tobin (1958) mostra que a estimação de um modelo de regressão com variável dependente censurada pelo método de mínimos quadrados ordinários é inapropriada, propondo uma metodologia alternativa que ficou conhecida como modelo de regressão censurada ou, simplesmente, modelo Tobit ${ }^{3}$.

A amostra compreende 67 firmas japonesas da indústria de transformação que entraram no país entre as décadas de 50 e 80, segundo JETRO (1992), com participação acionária superior a $10 \%$ em firmas brasileiras com unidades fabris instaladas e para as quais havia informações disponíveis para todas as variáveis incluídas no modelo.

O Quadro 1 resume as principais características da variável dependente.

3. Fundamentos teóricos a respeito do modelo Tobit podem ser encontrados em Tonooka (1998), que também apresenta outras referências bibliográficas sobre o tema.

12 TONOOKA, Eduardo Kiyoshi. Determinantes do Investimento Direto Japonês no Brasil... 
Quadro 1

Características da variável dependente segundo os setores de atividade

\begin{tabular}{lcc}
\hline Setor & $\begin{array}{c}\text { Número de } \\
\text { Empresas }\end{array}$ & $\begin{array}{c}\text { Média } \\
\text { (Desvio-Padrão) }\end{array}$ \\
\hline Produtos de Minerais Não-Metálicos & 1 & $0,03(-)$ \\
Metalurgia/Siderurgia & 6 & $0,25(0,18)$ \\
Mecânica & 13 & $0,12(0,12)$ \\
Material Elétrico e de Comunicações & 18 & $0,12(0,15)$ \\
Material de Transporte & 9 & $0,15(0,16)$ \\
Papel e Papelão & 1 & $0,23(-)$ \\
Química & 5 & $0,18(0,13)$ \\
Têxtil & 9 & $0,22(0,12)$ \\
Produtos Alimentares & 5 & $0,37(0,24)$ \\
Total & 67 & $0,18(0,16)$ \\
\hline
\end{tabular}

\subsection{Definição das variáveis explicativas}

A equação abaixo descreve a relação entre a variável dependente $\left(\mathrm{EMP}_{\mathrm{i}}\right)$ e as variáveis explicativas.

$$
\begin{array}{r}
\operatorname{EMP}_{\mathrm{i}}=\mathrm{a}_{1}+\mathrm{a}_{2} \operatorname{ANOS}_{\mathrm{i}}+\mathrm{a}_{3} \operatorname{POS}_{\mathrm{i}}+\mathrm{a}_{4} \cdot \text { P\&D }_{\mathrm{i}}+\mathrm{a}_{5} \operatorname{PROP}_{\mathrm{i}}+\mathrm{a}_{6} \cdot \operatorname{SAL}_{\mathrm{i}}+ \\
+\mathrm{a}_{7} \operatorname{RECNAT}_{\mathrm{i}}+\mathrm{a}_{8} \cdot \operatorname{CRESC}_{\mathrm{i}}+\mathrm{a}_{9} \cdot \operatorname{CONC}_{\mathrm{i}}+\mathrm{u}_{\mathrm{i}}
\end{array}
$$

A variável ANOS representa o número de anos que a empresa japonesa está instalada no país desde a sua constituição até o ano de 1989. Uma relação positiva com a variável dependente EMP sugere que as empresas instaladas há mais tempo apresentam maior interesse em manter negócios no país. Os conhecimentos, relacionamentos e experiência adquiridos ao longo da presença da empresa no país constituíram-se em verdadeiros ativos específicos à firma cuja utilização somente se demonstraria eficiente no próprio país em que foram desenvolvidos. Assim, ceteris paribus, o aproveitamento desses ativos justificaria uma atuação crescente da empresa junto ao mercado externo onde se criaram e se desenvolveram.

A variável POS é um índice que reflete o posicionamento da empresa no mercado japonês segundo o faturamento anual $($ Base $=1986)$. O ranking da empresa é ajustado pelo número total de empresas pertencentes ao seu setor de atividade segundo a fórmula:

$$
\mathrm{POS}_{\mathrm{i}}=\frac{\text { Número de empresas no setor }- \text { Ranking da empresa i no setor }+1}{\text { Número de empresas no setor }}
$$

Durante as décadas de 60 e 70, muitas empresas japonesas de pequeno e médio portes deslocaram suas atividades manufatureiras para o exterior devido à elevação dos custos de produção no Japão e ao acirramento da competição interna e externa. As empresas menores seriam as menos eficientes sob o ponto de vista produtivo e, 
consequientemente, as primeiras a sentir as dificuldades decorrentes da escassez crescente de matérias-primas e de mão-de-obra no país. Para essas empresas, as exportações tornavam-se menos competitivas mais rapidamente e a manutenção dos mercados externos exigia que a produção passasse a ser realizada em locais onde os fatores de produção fossem mais abundantes e baratos. Em algumas situações, a produção no exterior se destinava a terceiros mercados ou ao mercado japonês (caso de alguns países do Sudeste Asiático), enquanto que em outras a produção era absorvida, principalmente, pelo próprio mercado local (caso do Brasil). Assim, pode-se esperar uma relação negativa entre a variável dependente e a variável POS, ou seja, quanto melhor o posicionamento da empresa no mercado japonês, menor a importância relativa das atividades dessa empresa no Brasil.

A capacidade da firma para criar e desenvolver ativos específicos está representada, particularmente, pelas variáveis $\mathrm{P} \& \mathrm{D}$ e PROP, que significam, respectivamente, $\mathrm{O}$ montante de investimentos em pesquisa e desenvolvimento e em propaganda e marketing como proporção das vendas totais da firma no Japão. Essas variáveis têm a função de captar a importância dos ativos específicos (capacidade tecnológica, gerencial e de marketing, marca, reputação etc.) na decisão de investimento no exterior por parte da firma. Conforme os resultados encontrados em Pugel et alii (1996), a presença das empresas japonesas nos Estados Unidos mostra-se mais intensa nos setores em que o nível de investimentos em P\&D é mais elevado, induzindo a se esperar uma relação positiva da variável P\&D com a variável dependente EMP. Entretanto, a relação entre os gastos em P\&D e a importância relativa das atividades da firma em um determinado mercado não é tão óbvia.

Os gastos em P\&D geram ativos específicos que podem ser melhor explorados internacionalmente através do investimento direto e, neste sentido, pode-se esperar um maior grau de participação das afiliadas no exterior nas atividades totais das firmas com maior envolvimento em P\&D. A importância das atividades no exterior, consideradas como um bloco único vis-à-vis as atividades no mercado doméstico da matriz, deve relacionar-se positivamente com os gastos em $\mathrm{P} \& \mathrm{D}$, mas não se pode afirmar a existência desse mesmo tipo de relação no caso de se considerar apenas as atividades em um mercado externo específico, ainda mais se esse mercado não representar uma parcela significativa do total. Então, supondo que os mercados externos individuais (mercado de um país) tenham importâncias equivalentes ou que o aumento/diminuição das atividades no exterior seja distribuído proporcionalmente entre os mercados externos individuais, pode-se esperar um grau de associação positivo entre os gastos em P\&D e a variável dependente EMP.

O estabelecimento da relação esperada entre as variáveis PROP e EMP apresenta as mesmas dificuldades que as do caso anterior. Pugel et alii (1996) encontram uma associação positiva significativa entre gastos com propaganda e presença das empresas japonesas nos Estados Unidos. Yamawaki \& Audretsch (1988), em estudo sobre o efeito da diferenciação de produto sobre as importações de produtos japoneses pelos Estados Unidos, ressaltam que os gastos com propaganda realizados diretamente no mercado consumidor externo são a variável mais adequada para mensurar o grau de diferenciação dos produtos no exterior, pois levam em consideração as especificidades culturais de 
cada mercado. Dada a dificuldade para se obter esse tipo de informação e para poderem trabalhar com as estatísticas sobre gastos com propaganda disponíveis para o Japão, os autores sugerem como hipótese considerar que os gastos com propaganda sejam uma proporção constante das vendas em cada mercado. Por outro lado, Caves (1974) argumenta que os gastos com propaganda realizados pela matriz em seu mercado doméstico são a estatística relevante, pois constituem a base para a construção e consolidação de sua marca.

É razoável supor que, no caso brasileiro, a reputação dos produtos de empresas japonesas origina-se, basicamente, do sucesso alcançado por essas empresas no mercado mundial e que os gastos com propaganda no país têm uma contribuição marginal para reforçar essa imagem. Assim, a utilização dos dados relativos aos gastos com propaganda no Japão parecem ser os mais indicados, independentemente da disponibilidade ou não dessas informações para as afiliadas brasileiras, para se avaliar o grau de participação das empresas japonesas nos diversos setores da indústria nacional. Já para se analisar a importância relativa das atividades no Brasil, as mesmas observações feitas em relação aos gastos em P\&D são pertinentes para o caso dos gastos com propaganda, a partir das quais se pode prever uma associação positiva entre esta variável e a variável dependente EMP.

A variável SAL foi obtida através da divisão do salário mensal médio setorial em dólar no Brasil (IBGE 2 dígitos) e do salário mensal médio pago pela firma em dólar no Japão. Assim, definiu-se um indicador para as diferenças de custos com mão-de-obra existentes entre os dois países. De acordo com a argumentação baseada nos custos comparativos, empresas de países onde a mão-de-obra é fator escasso (Japão) deslocariam sua produção para países onde esse fator é abundante (Brasil). É de esperar, portanto, uma relação negativa entre esta variável e a variável dependente EMP.

A variável RECNAT é uma variável dummy destinada a identificar a influência da escassez de determinados recursos naturais no Japão sobre a decisão de investir no Brasil. Para as empresas dos setores de papel e celulose, siderurgia básica, pescado, bem como as processadoras de fibras naturais que se instalaram durante a década de 70 , a variável RECNAT assume o valor " 1 "; caso contrário, assume o valor "0"

A variável CRESC representa a taxa média de crescimento da produção física do setor em que atua a empresa no Brasil durante a década de 80 (segundo a classificação do IBGE para a matriz de relações intersetoriais - Matriz 100), obtida a partir da estimação de equações de regressão semi-logarítmicas para cada um dos setores relevantes. Esta variável é empregada como proxy para identificar o tamanho e o potencial de crescimento do mercado da empresa no Brasil, devendo apresentar uma relação positiva com a variável dependente EMP. Rigorosamente, o efeito do crescimento do mercado brasileiro deveria ser compensado pelo crescimento do próprio mercado japonês e de terceiros mercados, ou seja, o emprego de um fator de crescimento relativo dos mercados seria o procedimento mais adequado nesta situação. Dada a limitação dos dados, todavia, são incluídas apenas as taxas de crescimento absoluto referentes ao Brasil, o que pode prejudicar os resultados da estimação do parâmetro correspondente.

A variável CONC representa o grau de concentração industrial (CR 4), segundo as vendas totais das empresas, prevalecente no ano de 1980, estimado por Willmore 
(1987) a partir do cadastro de informações tributárias da Secretaria da Receita Federal. A relação esperada com a variável dependente EMP é ambígua, pois nos setores mais concentrados as barreiras à entrada são mais severas (impacto negativo), mas a expectativa de retorno é maior (impacto positivo). Como no período sob análise a maior parte das empresas consideradas já estava instalada no país, espera-se que a segunda influência prevaleça, proporcionando uma relação positiva entre a variável CONC e a variável dependente EMP.

O Quadro 2 sintetiza o significado das variáveis utilizadas no modelo.

\section{Quadro 2}

Descrição sucinta das variáveis

\begin{tabular}{ll}
\hline EMP $(0,18 ; 0,16)$ & $\begin{array}{l}\text { Número de empregados no Brasil / Número de empregados no } \\
\text { Japão }(1989) \\
\text { Número de anos que a empresa está instalada no país (Base } \\
1989)\end{array}$ \\
ANOS $(18,46 ; 7,24)$ & $\begin{array}{l}\text { Posição relativa da empresa no mercado japonês segundo } \\
\text { faturamento }(1986)\end{array}$ \\
POS $(0,81 ; 0,22)$ & $\begin{array}{l}\text { Investimentos em P\&D / Vendas da empresa no Japão (Média } \\
1987-1989)\end{array}$ \\
P\&D $(3,86 ; 2,48)$ & $\begin{array}{l}\text { Gastos com propaganda / Vendas da empresa no Japão (Média } \\
1985-1987)\end{array}$ \\
PROP $(0,85 ; 1,16)$ & $\begin{array}{l}\text { Salário médio setorial no Brasil / Salário médio da empresa } \\
\text { no Japão (1989) }\end{array}$ \\
SAL $(0,21 ; 0,05)$ & $\begin{array}{l}\text { Dummy para identificar os setores intensivos em recursos } \\
\text { naturais } \\
\text { Crescimento médio da produção física do setor no Brasil (1981- } \\
\text { RECNAT }(0,15 ; 0,36)\end{array}$ \\
CRESC $(4,26 ; 1,97)$ & $\begin{array}{l}1989) \\
\text { Grau de concentração do setor no Brasil (1980) }\end{array}$ \\
\hline
\end{tabular}

Notas: 1. Valores entre parênteses representam a média e o desvio-padrão das variáveis, respectivamente.

2. Para a descrição completa das fontes de dados, ver Tonooka (1998).

\subsection{Resultados da estimação do modelo}

A equação da página seguinte expõe os principais resultados da estimação do modelo de regressão linear a partir do método Tobit.

Dado que o objetivo do modelo é identificar os determinantes do investimento japonês no Brasil ao nível das firmas, a técnica de cross-section apresenta restrições para a avaliação dos impactos das variáveis de caráter macroeconômico. Esta limitação decorre do fato dessas variáveis assumirem um mesmo valor para todas as firmas (por exemplo, valor do PIB, taxa de crescimento do PIB, nível de inflação, taxa de juros, 


$$
\begin{aligned}
& \mathrm{EMP}_{\mathrm{i}}=0,1855+0,0073 \quad \mathrm{ANOS}_{\mathrm{i}}-0,2873 \quad \mathrm{POS}_{\mathrm{i}}-0,0193 \quad \mathrm{P}_{\mathrm{AD}}+ \\
& \begin{array}{llll}
(0,1275) & (0,0026)^{\mathrm{a}} & (0,0839)^{\mathrm{a}} & (0,0082)^{\mathrm{b}}
\end{array} \\
& +0,0519 \mathrm{PROP}_{\mathrm{i}}+0,2979 \mathrm{SAL}_{\mathrm{i}}+0,1074 \mathrm{RECNAT}_{\mathrm{i}}+ \\
& (0,0145)^{\mathrm{a}} \quad(0,3800) \quad(0,0486)^{\mathrm{b}} \\
& +0,0124 \text { CRESC }_{\mathrm{i}}-0,0248 \mathrm{CONC}_{\mathrm{i}} \\
& (0,0113) \quad(0,0811)
\end{aligned}
$$$$
\mathrm{R}^{2}=0,42 \quad \mathrm{~F}=5,25 \quad \mathrm{~F}(\text { teste White })=0,97 \quad \mathrm{n}=67
$$

Notas: 1. Valores entre parênteses representam os erros-padrão.

2. Letras "a" e "b" indicam coeficientes diferentes de zero aos níveis de significância de $1 \%$ e

$5 \%$, respectivamente (teste bicaudal).

taxa de câmbio etc.), muito possivelmente com efeitos diferenciados sobre cada uma delas, impossibilitando a estimação do modelo caso fossem incluídas ${ }^{4}$.

A equação revela que os coeficientes associados às variáveis que procuram captar a importância dos ativos específicos à firma (P\&D e PROP) apresentam-se significativamente diferentes de zero. Entretanto, o coeficiente associado à variável $\mathrm{P} \& \mathrm{D}$ exibe sinal oposto à expectativa inicial. No caso, a presença dos ativos específicos criados pelos investimentos em P\&D (particularmente, tecnologia) não influenciaria a decisão da empresa japonesa em investir no Brasil. Ao contrário, as empresas com menor parcela de recursos destinados às atividades de $\mathrm{P} \& \mathrm{D}$ é que apresentariam uma maior atuação no país. $\mathrm{O}$ coeficiente associado à variável PROP, no entanto, apresenta sinal de acordo com o esperado, demonstrando que a marca e a reputação dos produtos e empresas japonesas agem favoravelmente na presença dessas empresas no país ${ }^{5}$

$\mathrm{O}$ fato de o coeficiente associado à variável POS mostrar-se significativo e com o sinal esperado reforça o argumento da presença de empresas japonesas de menor porte em países com menor pressão concorrencial (do Sudeste Asiático e da América Latina, especificamente). Este resultado mais a suposição de que os investimentos em P\&D estão diretamente associados com o tamanho da empresa fazem crer que a relação inversa entre a variável dependente e a variável P\&D seja coerente para o caso analisado, muito

4. De certo modo, parte do efeito das variáveis de caráter macroeconômico poderia estar sendo captado pela presença da constante no modelo. A constante representa o componente "autônomo" da variável dependente, ou seja, que não é explicado pelas variáveis incluídas no modelo e que não está sujeito às variações randômicas entre as firmas (portanto, não presente no termo aleatório). Assim, para que a constante pudesse estar associada a alguma variável "macro" seria necessário supor que todas as firmas avaliassem igualmente essa variável na sua decisão de investimento, sem dúvida, uma hipótese muito forte. Para se verificar a influência dessas variáveis sobre a decisão de investimento das firmas, então, seria necessário empregar técnicas de série de tempo ou de painel, envolvendo mais de um país na análise.

5. Quando os dados para as variáveis P\&D e PROP foram ajustados para levarem em consideração a existência de diferenças nos níveis médios de gastos com P\&D e com propaganda e marketing entre as indústrias não houve mudanças nos resultados. Apenas a constante passou a apresentar significância ao nível de 5\%, enquanto que os coeficientes estatisticamente não-nulos da equação mantiveram seus sinais e níveis de significância. Para maiores detalhes, ver Tonooka (1998). 
embora, em termos estatísticos, a existência de correlação entre as variáveis POS e $P \& D$ possa comprometer a qualidade das estimativas dos seus coeficientes ${ }^{6}$

Já os resultados apresentados pela variável ANOS, por sua vez, ratificam a idéia de que uma presença mais prolongada no Brasil contribui para a expansão ou manutenção das atividades da empresa no país. Nessas situações, pode-se estar pretendendo uma maior utilização dos ativos específicos localmente desenvolvidos ou, apenas, a sua simples preservação para o caso de as empresas estarem aguardando por um momento mais atraente para a ampliação das suas atividades no país. Segundo estudo do MCT (1993), as afiliadas de empresas japonesas instaladas no país têm apresentado indicadores de rentabilidade sobre o investimento realizado inferiores às suas congêneres de outras nacionalidades. A pequena importância relativa das atividades no Brasil reduz os custos de se operar no prejuízo ou com margens insignificantes no país. Assim, é factível supor que para a maior parte das empresas japonesas instaladas no país tem prevalecido o caráter inercial do investimento ${ }^{7}$

O coeficiente associado à variável SAL mostra-se com sinal oposto ao inicialmente previsto, mas estatisticamente não-significativo, implicando que as diferenças salariais entre os países não influenciam o grau de presença das empresas japonesas no Brasil. Esse resultado sugere que as empresas japonesas com maior atuação no país pertencem aos setores em que as diferenças salariais são menores, revelando que essas empresas não estão primordialmente interessadas em explorar vantagens de custos associados à mão-de-obra, que parece ser uma preocupação mais presente nos investimentos direcionados ao Sudeste e Leste Asiáticos ${ }^{8}$.

O coeficiente da variável RECNAT mostra-se significativo e com o sinal esperado, demonstrando a importância, ainda, na década de 80 , dos investimentos com o objetivo de garantir o acesso a fontes estáveis e mais baratas de produtos básicos e intermediários no Brasil.

Apesar de estatisticamente não-significativo, o coeficiente associado à variável CRESC apresenta sinal positivo, conforme a expectativa inicial. O coeficiente associado à variável CONC, também não-significativo, possui sinal negativo, ou seja, a atuação das empresas japonesas no país mostra-se mais importante nos setores menos concentrados.

Em síntese, verificou-se que as variáveis no nível "meso" (setorial) não se mostraram significativas para explicar a presença das empresas japonesas no país durante o período analisado. Esse resultado não é surpreendente haja vista o desempenho apresentado pela economia brasileira em toda a década de 80. Assim, conforme era a

6. A correlação entre as variáveis POS e P\&D no modelo é baixa $(0,31)$, não devendo comprometer sobremaneira os resultados.

7. Hufbauer et alii (1994), investigando os determinantes da distribuição dos fluxos de investimento direto estrangeiro de Alemanha, Estados Unidos e Japão, verificam que, no caso japonês apenas, o estoque de investimento já existente no país recebedor influencia significativamente o fluxo de investimento novo a ser direcionado àquele país. Os autores atribuem este resultado ao "conservadorismo" das firmas japonesas, que "investem hoje onde investiram ontem"

8. Liu et alii (1997), por exemplo, mostram o efeito significativo dos baixos salários na China sobre as decisões de investimento direto estrangeiro neste país no período 1983-1994. 
expectativa preliminar deste estudo, foram as variáveis em nível "micro" (da firma) que responderam mais firmemente pelas diferenças nos graus de envolvimento das empresas japonesas com o país.

Quase todas as variáveis que procuraram captar diferenças entre as firmas japonesas que investem no país (ANOS, POS, P\&D, PROP) mostraram coeficientes estatisticamente diferentes de zero. Os sinais e a significância dos coeficientes associados às variáveis ANOS e PROP revelam a importância da presença de certos ativos específicos à firma. $\mathrm{O}$ resultado apresentado pela variável $\mathrm{POS}$ ressalta o fato das firmas médias $\mathrm{e}$ pequenas atuarem com mais intensidade nos mercados dos países em desenvolvimento. Já os resultados obtidos para as variáveis P\&D e SAL, esta com coeficiente estatisticamente não-significativo, indicam algumas particularidades das firmas japonesas em relação ao mercado brasileiro, as quais não se instalaram no país com o objetivo precípuo de obter vantagens de custos para o estabelecimento de bases de exportação.

\section{Considerações Finais}

Durante a década de 70, o IDE japonês representou uma estratégia de relocalização de indústrias em declínio (sunset industries). Desta maneira, esse investimento direcionou-se essencialmente à produção no exterior de bens nos quais o país perdia a sua competitividade internacional. Ásia e América Latina (Brasil, em especial) foram os principais recebedores do IDE japonês que se concentrou nos setores manufatureiro (químico, têxtil, siderúrgico, material elétrico) e extrativo-mineral.

A partir dos anos 80, verificou-se uma mudança, tanto em nível espacial quanto setorial, na composição do IDE japonês. Estados Unidos e Europa aumentaram sua participação no total investido pelo Japão no exterior, enquanto ampliou-se a parcela de investimentos destinados aos setores financeiro e imobiliário. No setor manufatureiro, observou-se uma realocação de recursos em direção aos segmentos mais intensivos na utilização de capital e tecnologia, como o automobilístico e o eletrônico.

$\mathrm{Na}$ década seguinte, o Japão passou a enfrentar graves problemas internos decorrentes da má administração de suas políticas monetária e financeira que limitaram a capacidade de investimento de suas firmas no exterior. Após atingir seu ápice em 1990 e declinar sucessivamente até 1993, o nível do investimento direto japonês vem aumentando gradualmente desde então. Do outro lado, o Brasil passou a ser um dos principais recebedores de investimento direto dentre os países em desenvolvimento a partir de 1996 à medida que a estabilização monetária e a implementação de reformas estruturais no sistema econômico, particularmente, no tocante às privatizações, demonstravam avanços.

Entretanto, com as mudanças estruturais apresentadas pelo IDE japonês desde a década de 80, o Brasil viu diminuída sua importância para as empresas japonesas que, em se tratando das economias em desenvolvimento, passaram a direcionar seus recursos preferencialmente para os países do Sudeste Asiático e, mais recentemente, para a China. Como consequiência, também diminuiu o interesse relativo por parte de estudiosos e pesquisadores sobre as relações de investimento entre Japão e Brasil. 
A análise empírica mostrou que as diferenças de custos com mão-de-obra entre os países não influenciam o grau de presença das empresas japonesas no Brasil. Pelo contrário, os resultados demonstraram que as empresas com maior atuação no país pertencem aos setores nos quais essas diferenças são menores, sugerindo que essas empresas não estão primordialmente interessadas em explorar vantagens de custos associados à mão-de-obra, que parece ser uma preocupação mais presente nos investimentos na Ásia. Também não significativas foram as características dos setores em que atuam as empresas, como o grau de concentração e a taxa de crescimento do setor.

O tamanho da firma no Japão é um fator que influencia a presença das firmas japonesas no Brasil, confirmando a argumentação de que há a ocorrência de firmas japonesas de menor porte nos países que não apresentam pressões concorrenciais tão severas quanto no Japão.

Finalmente, o tempo decorrido desde a instalação da empresa japonesa no Brasil mostrou-se um fator relevante para explicar a presença dessas empresas no país. A manutenção ou expansão das atividades de uma determinada empresa no país podem ser o resultado da maior utilização dos ativos específicos localmente desenvolvidos ou, apenas, da sua simples preservação para o caso de as empresas estarem aguardando por um momento mais atraente para a ampliação das suas atividades no país. Neste sentido, consideramos que para a maior parte das empresas japonesas instaladas no país prevaleceu o caráter inercial do investimento no período considerado.

\section{Bibliografia}

CAVES, Richard. "Causes of direct investment: foreign firms shares in Canadian and United Kingdon manufacturing industries" The Review of Economics and Statistics. 56(3), 1974, pp. 279-293.

HUFBAUER, Gary et alii. "Determinants of direct foreign investmen and its connection to trade". UNCTAD Review. 1, 1994, pp. 39-51.

JETRO. Nikkei shinshutsu kigyô kaiyô dôtôshi saki kigyô risuto. São Paulo, JETRO, 1992.

LIU, Xiaming. et alii. "Country characteristics and foreign direct investment in China: a panel data analysis" Weltwirtschafiliches Archiv. 133(2), 1997, pp. 313-329.

MCT. Estudo da Competitividade da Indústria Brasileira: Oportunidades Abertas para o Brasil Face aos Fluxos Globais de Investimento de Risco e de Capitais Financeiros nos Anos 90. Campinas, Ministério da Ciência e Tecnologia, 1993.

PUGEL, Thomas et alii. "Further evidence on Japanese direct investment in US manufacturing" The Review of Economic and Statistics. 78(2), 1996, pp. 208-213.

TOBIN, James. "Estimation of relationships for limited dependent variables". Econometrica. 26(1), 1958, pp. 24-36.

TONOOKA, Eduardo. Investimento Direto Japonês no Brasil na Década de 80: Uma Análise de seus Determinantes no Brasil e no Mundo. São Paulo, FEA/USP, tese de doutorado, 1998.

WILlMORE, Larry. "Controle Estrangeiro e Concentração na Indústria Brasileira” Pesquisa e Planejamento Econômico. 17(1), 1987, pp. 161-190. 
YAMAWAKI, Hideki \& AUDRETSCH, David. "Import share under international oligopoly with differentiated products: Japanese imports in US manufacturing". The Review of Economics and Statistics. 70(4), 1988, pp. 569-579.

Endereço para correspondência: Research Institute for Economics and Business Administration

Kobe University Rokkodai-cho Nada-ku Kobe-shi 657-8501 Japan Tel/Fax: (81)78-803-0403 